\title{
Stegemann, Thomas
}

\section{Lieder in der Musiktherapie mit Kindern}

Praxis der Kinderpsychologie und Kinderpsychiatrie 56 (2007) 1, S. 40-58

Quellenangabe/ Reference:

Stegemann, Thomas: Lieder in der Musiktherapie mit Kindern - In: Praxis der Kinderpsychologie und Kinderpsychiatrie 56 (2007) 1, S. 40-58 - URN: urn:nbn:de:0111-opus-30442 - DOI: 10.25656/01:3044

\section{Vandenhoeck \& Ruprecht}

http://www.v-r.de

\section{Nutzungsbedingungen}

Gewährt wird ein nicht exklusives, nicht übertragbares, persönliches und beschränktes Recht auf Nutzung dieses Dokuments. Dieses Dokument ist ausschließlich für den persönlichen, nicht-kommerziellen Gebrauch bestimmt. Die Nutzung stellt keine Übertragung des Eigentumsrechts an diesem Dokument dar und gilt vorbehaltlich der folgenden Einschränkungen: Auf sämtlichen Kopien dieses Dokuments müssen alle Urheberrechtshinweise und sonstigen Hinweise auf gesetzlichen Schutz beibehalten werden. Sie dürfen dieses Dokument nicht in irgendeiner Weise abändern, noch dürfen Sie dieses Dokument für öffentliche oder kommerzielle Zwecke vervielfältigen, öffentlich ausstellen, aufführen, vertreiben oder anderweitig nutzen.

Mit der Verwendung dieses Dokuments erkennen Sie die Nutzungsbedingungen an.

\section{Terms of use}

We grant a non-exclusive, non-transferable, individual and limited right to using this document.

This document is solely intended for your personal, non-commercial use. Use of this document does not include any transfer of property rights and it is conditional to the following limitations: All of the copies of this documents must retain all copyright information and other information regarding legal protection. You are not allowed to alter this document in any way, to copy it for public or commercial purposes, to exhibit the document in public, to perform, distribute or otherwise use the document in public.

By using this particular document, you accept the above-stated conditions of use.

\section{Kontakt / Contact:}

\section{DeDOCS}

DIPF | Leibniz-Institut für Bildungsforschung und Bildungsinformation Informationszentrum (IZ) Bildung

E-Mail: pedocs@dipf.de

Internet: www.pedocs.de

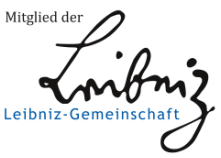




\title{
ÜBERSICHTSARBEITEN
}

\section{Lieder in der Musiktherapie mit Kindern}

\author{
Thomas Stegemann
}

\section{Summary}

Songs in music therapy with children

For most of us, songs and nursery rhymes have a special emotional quality and, thus, are part of the basic repertoire of a music therapist. This paper outlines the meaning and applicability of songs in music therapy with children. The first part discusses the significance of songs within the context of developmental psychology, referring to the development of basic psychological functions such as motorical skills, language, cognition, emotion, mental representations, motivation and intention. The second part deals with indications and objectives as well as different applications of songs in music therapy. Traditional and new nursery rhymes, free renderings, playing songs, spontaneous tunes, welcome and farewell songs and semi-structured tunes are introduced. Finally, the article reviews the diverse therapeutical functions of songs within the process of music therapy as well as essential qualifications for a music therapist.

Prax. Kinderpsychol. Kinderpsychiat. 56/2007, 40-58

\section{Keywords}

music therapy with children - developmental psychology - songs - child- and adolescent psychiatry

Kinderlieder haben für die meisten Menschen eine besondere emotionale Bedeutung und gehören zum grundlegenden Handwerkszeug eines Kindermusiktherapeuten. Dieser Artikel erörtert die Bedeutung und Einsatzmöglichkeiten von Liedern in der Kindermusiktherapie. Im ersten Teil wird die Funktion von Liedern im entwicklungspsychologischen Kontext diskutiert. Dies geschieht in Bezugnahme auf die für die Entwicklung der basalen psychischen Funktionen relevanten Bereiche: Motorik, Sprache, Kognition, Emotion, mentale Repräsentationen sowie Motivation und Intention. Im zweiten Teil werden sowohl unterschiedliche Indikationen und Zielsetzungen als auch die verschiedenen Einsatzmöglichkeiten von Liedern in der musiktherapeutischen Praxis behandelt. Dabei geht es um klassische und aktuelle Kinderlieder, die Liedform der Kontrafaktur, Spiel- und Situationslieder, das Ritual des Begrüßungs- und Abschiedsliedes und um semistrukturierte Lieder, die sich als projektives Diagnostikum einsetzen lassen. Abschließend wird auf die vielfältigen therapeutischen Funktionen des Liedes innerhalb des musiktherapeutischen Prozesses und auf die notwendigen Voraussetzungen auf therapeutischer Seite hingewiesen. 


\section{Schlagwörter}

Kindermusiktherapie - Entwicklungspsychologie - Lieder - Kinder- und Jugendpsychiatrie

Wir brauchen Musik. Das Gespenst ist die lautlose Welt. Ingeborg Bachmann

Lieder aus der Kindheit haben für die meisten Menschen eine besondere emotionale Bedeutung. So ist für den Autor dieses Artikels eine der frühesten Kindheitserinnerungen mit dem Lied „Hänschen klein“ und einer der ersten Autonomiebestrebungen - einem „unerlaubten“ Ausflug zu einem nahe gelegenen Bauernhof - verbunden: Im zarten Alter von vielleicht zweieinhalb Jahren diente das fröhlich vor sich her gesungene Kinderlied als Drehbuch („ging allein in die weite Welt hinein“) sowie vermutlich auch als Mutmacher („Stock und Hut steht ihm gut, ist gar wohlgemut“) für das erste einsame Abenteuer, allerdings ohne bekanntes Happy-End („da besinnt sich das Kind, läuft nach Haus geschwind“), da ihn die Mutter erst aus einem Kuhstall abholen musste, wo er gerade ausprobierte, was sich für interessante Töne aus abgefallenen Kuhhörnern entlocken lassen.

Laut Plahl und Koch-Temming (2005) zählt der „systematische Einsatz von Liedern in der Musiktherapie mit Kindern [...] zum grundlegenden Handwerkszeug einer Kindermusiktherapeutin“ (S. 179). Dabei sind es nicht nur „klassische“ Kinderlieder, die zum Einsatz kommen; das Repertoire umfasst vielmehr ein breites Spektrum, welches von liedartigen Improvisationen über alte und neuere Kinderlieder bis hin zur Popularmusik der „Erwachsenenwelt“ reicht. Dieser Artikel soll einen Ein- und Überblick hinsichtlich verschiedener Anwendungsbereiche von Liedern in der musiktherapeutischen Arbeit mit Kindern geben und dies anhand kurzer Fallvignetten verdeutlichen. Einleitend soll im ersten Teil auf die Bedeutung von (Kinder-)Liedern aus entwicklungspsychologischer Perspektive eingegangen werden.

\section{Entwicklungspsychologische Aspekte}

Die Wahrnehmung von Musik beginnt schon pränatal im Mutterleib. Der erste Kontakt - im wortwörtlichen, also taktilen Sinn - findet vermutlich über den Tastsinn der Haut statt, der bereits vor dem Hörsinn genügend entwickelt ist, um Schallwellen zu registrieren. Von einer akustischen Wahrnehmung von Geräuschen, Tönen, Sprache und Musik kann man etwa ab der 28. Schwangerschaftswoche ausgehen (Spitzer 2002b). Von diesem Zeitpunkt an findet die musikalische Sozialisation statt, die den werdenden Menschen mit den wichtigsten musikalischen Parametern vertraut macht (Decker-Voigt 1999):

(c) Vandenhoeck \& Ruprecht GmbH \& Co. KG, Göttingen 
- mit dem Rhythmus, der sich über den Herzschlag und das Ein- und Ausatmen der Mutter vermittelt,

- mit Klang und Melodie, welche in den Körpergeräuschen (Gelenke, Darm etc.) und Körpertönen (Herzschlag und vor allem Stimme der Mutter) enthalten sind, die aber zunehmend auch von außen an das Kind herangetragen werden (Alltagsgeräusche, Stimme des Vaters, Musik aus der Umgebung etc.)

- und mit der Dynamik, welche die Gegensätze in Tempo, Lautstärke und Tonhöhe der oben genannten Merkmale beinhaltet und erlebbar werden lässt.

Welch (2005) weist darauf hin, dass sich im letzten Schwangerschaftstrimester parallel zum auditorischen System auch die für das Erleben von affektiven Zuständen notwendigen Strukturen und Funktionen auf nervöser, endokriner und immunologischer Ebene ausbilden. Dies bedeutet, dass beispielsweise die Vokalisationen der Mutter - mit dem entsprechenden emotionalen Korrelat einhergehend - zu einer gleichsinnigen neuroendokrinen Reaktion bei dem sich entwickelnden Kind führen. Das erklärt, warum Neugeborene von Beginn an die musikalischen Botschaften verstehen, „die ihre Eltern und Bezugspersonen intuitiv überall auf der Welt verwenden, um mit ihrem Baby zu kommunizieren“ (Plahl u. Koch-Temming 2005). Mechthild Papoušek (2003) schreibt in diesem Zusammenhang: „Mit gewissem Recht wird die vorsprachliche Kommunikation zwischen Eltern und Kind in der Literatur immer wieder mit musikalischen Metaphern umschrieben, als ,gemeinsamer Tanz', als ,Duett' oder ,Unisono-Gesang. [...] Musikalische Elemente werden bald zu einem ersten gemeinsamen Alphabet der vorsprachlichen Kommunikation und zu einem gemeinsamen Medium, das wechselseitiges Entrainment, affektive Abstimmung und Austausch emotionaler Erfahrung und Bewertung des Erlebten ermöglicht. Gleichzeitig dienen die musikalischen Elemente auch verschiedenen linguistischen Funktionen in Vorbereitung auf den Spracherwerb" (S. 8).

Resch et al. (1999) beschreiben in der Entwicklung der basalen psychischen Funktionen folgende Bereiche: Motorik, Sprache, Kognition, Emotion, mentale Repräsentationen sowie Motivation und Intention. Auf alle diese Domänen haben (Kinder-)Lieder einen wichtigen Einfluss, wobei unter Kinderliedern sowohl Lieder von Kindern als auch Lieder für Kinder verstanden werden sollen.

\subsection{Motorik}

Nach Resch et al. (1999) ist „die motorische Entwicklung insofern für die psychische Entwicklung von Bedeutung, als die persönliche ,Eroberung' des Erfahrungsraumes, das Kennenlernen von Gegenständen und das interaktive Spiel mit Bezugspersonen funktionstüchtige motorische Abläufe benötigt" (S. 118). Wie sich anhand von Bewegungsstörungen (z.B. Parkinson oder ADHD) zeigen lässt, ist der reguläre Ablauf von Bewegungen u.a. abhängig von Timing und Rhythmus, das heißt von grundlegenden musikalischen Parametern, deren Vermittlung wie oben dargestellt

(C) Vandenhoeck \& Ruprecht GmbH \& Co. KG, Göttingen 
schon pränatal beginnt. Lieder erhalten hier insbesondere in Form von Tanz- und Bewegungsliedern eine wichtige Funktion. Die Musik wirkt dabei funktionell als „basaler Sinnesstimulus“ und als „Integrator“ (Frohne-Hagemann u. Pleß-Adamczyk 2005). Im ersten Fall dienen „Klang-, Rhythmus-, Intensitäts- und Formerfahrungen der sensorischen (intermodalen) Integration und der Verbesserung der neurophysiologischen Wahrnehmungs-, Verarbeitungs- und Handlungszyklen“" (Frohne-Hagemann u. Pleß-Adamczyk 2005, S. 97). In zweiten Fall kann Musik in ihrer integrierenden Funktion z.B. einen Zusammenhang zwischen Sprache und Handlung herstellen und vermitteln helfen. „Diese Funktion hat große Bedeutung, wenn es für das Spiel eines Kindes wichtig ist, dass es durch die Musik Sinnzusammenhänge erlebt“" (Frohne-Hagemann u. Pleß-Adamczyk 2005, S. 98).

\subsection{Sprache}

Die Beeinflussung von Musik und Sprache scheint eine wechselseitige zu sein. Zum einen sind musikalische Elemente unverzichtbarer Bestandteil der Sprache (z.B. Sprachmelodie, Sprechrhythmus oder Stimmhöhe), zum anderen erwachsen die Anregungen für die Entwicklung musikalischer Fähigkeiten aus den sprachlichen und nichtsprachlichen Vokalisationen der Bezugspersonen (Bruhn u. Oerter, 1997). Wie die Autoren weiter ausführen, ist das Entscheidende dabei, dass die Bezugspersonen ihre Sprechweise an die Bedürfnisse und Fähigkeiten des Neugeborenen anpassen, was kulturübergreifend intuitiv geschieht (S. 276):

- Die Sprachmelodien sind von der Kontur (dem Auf und Ab der Tonhöhe) her einfach strukturiert.

- Der benutzte Stimmumfang ist deutlich größer als in der Kommunikation mit Erwachsenen.

- Die Melodiestrukturen werden oft wiederholt und sind bei ähnlichen Aussagen identisch.

- Das Repertoire an Melodie-Prototypen ist begrenzt.

All das erleichtert es dem Säugling, diese Vorgaben stimmlich nachzuahmen. Im Alter von zwei bis drei Monaten ist die erste Veränderung der Grundfrequenz der Stimme nachweisbar (Fox 1990). Während dem Kind in den ersten Monaten nur eine beschränkte Auswahl von Lauten zur Verfügung steht (k, g, x, i, a), kommt es etwa ab einem halben Jahr - in der so genannten Lallperiode - zu komplexeren Lautverbindungen (Resch et al. 1999). Wie Plahl und Koch-Temming (2005) beschreiben, entwickelt sich das kindliche Experimentieren mit Stimmlauten allmählich zum „spontanen Singen“. Erste einfache Formen in der Struktur dieser Stimmspiele lassen sich bereits ab dem Alter von etwa eineinhalb Jahren erkennen. „Mit wachsenden sprachlichen Fähigkeiten singt das Kind immer häufiger ohne Aufforderung spontan Lieder, in denen es seine Erlebnisse in erfundenen improvisierten Liedern schildert, oder bekannte Lieder singt und diese nach eigenen Vorstellungen variiert"

(c) Vandenhoeck \& Ruprecht GmbH \& Co. KG, Göttingen 
(Plahl u. Koch-Temming 2005, S. 29). Auf diese Weise fördern sich Sprachentwicklung und musikalische Aktivität gegenseitig, und insbesondere Lieder führen zu einer Erweiterung von Ausdrucksmöglichkeiten, Sprachschatz und Formverständnis.

\subsection{Kognition}

Nicht umsonst dienen Reime („Trenne nie ,st', denn es tut ihm weh“) oder Lieder („A, B, C, D, E: hier kommt die Buchstabenfee“ [Detlef Cordes]) schon immer als „rhythmische Lernhilfen“ zum Erlernen und Erinnern von Buchstaben, Zahlen, grammatikalischen oder orthographischen Regeln. Nicht zuletzt durch Untersuchungen zum so genannten „Mozart-Effekt“ (Rauscher u. Shaw 1998) und infolge einer sechsjährigen Langzeitstudie zur Wirkung von zusätzlichem Musikunterricht an Berliner Grundschulen (Bastian 2002) wurden die positiven Effekte von Musik auf kognitive Funktionen wieder ins Gedächtnis gerufen. „Wieder“ deshalb, weil - vermutlich auf Grundlage einschlägiger Erfahrungen - die musikalische Bildung im antiken Griechenland ebenso zu den „Grundlagen-Wissenschaften“ gehörte, wie sie bis zum Beginn der Neuzeit selbstverständlicher Teil z.B. des Medizinstudiums war.

Wie dauerhaft gerade Liedtexte im Gedächtnis verankert sind, wird eindrucksvoll deutlich beim Singen und Musizieren mit demenzkranken Patienten, denen zwar große Bereiche ihres Erinnerungsvermögens abhanden gekommen sind, die aber nicht selten ohne Probleme mehrstrophige Volks- und Kirchenlieder auswendig singen können. Wie und auf welcher Ebene des Gedächtnisapparates die Musik als „mnemotechnisches Doping“ wirkt, ist letztlich nicht geklärt. Sicher ist nur, dass Informationen, die in einem positiven emotionalen Kontext erworben wurden, am besten erinnert werden können (Erk et al. 2003). Dies scheint neben Rhythmus und Wiederholung der Hauptmechanismus für die gedächtnisfördernde Wirkung von Musik zu sein, da Singen und Musizieren in den meisten Fällen mit emotional positiv besetzten Erlebnissen verbunden wird. Insgesamt ist der Zusammenhang zwischen Kognition und Musik aber aufgrund seiner Komplexität noch nicht gut verstanden und wenig wissenschaftlich untersucht, was insbesondere für den Bereich der Entwicklung im Kindes- und Jugendalter gilt.

\subsection{Emotion}

Die Verbindung zwischen Musik und Emotionen ist eine grundlegende. So sind es im Wesentlichen musikalische Elemente, die dem gesprochenen Wort eine emotionale Färbung geben. In diesem Zusammenhang mag es nicht verwundern, dass Kinder den emotionalen Gehalt von Musik viel früher verstehen als den von Sprachmelodie, wie in einer Studie von Doherty et al. (1999) gezeigt werden konnte. Ein gutes Beispiel für die enge und frühe Beziehung zwischen Musik und Emotionen sind Wiegenlieder, die nach Spitzer (2002b) „gewissermaßen zwischen Musik einerseits und stark mit positiven, liebenden, beruhigenden Emotionen geladener Sprache

(C) Vandenhoeck \& Ruprecht GmbH \& Co. KG, Göttingen 
andererseits" stehen (S. 386). Später in der Entwicklung wird die Musik, wie Plahl und Koch-Temming (2005) beschreiben, ein wichtiges Medium für das Kind, um sich mitzuteilen und Beziehungen zu anderen herzustellen. Dies geschieht neben dem spontanen Singen durch das gemeinsame Singen von Kinderliedern ebenso wie durch Kinderspiele und -tänze. Dies könnte eine Erklärung dafür bieten, warum für Kinder die musikalische Darstellung unterschiedlicher Emotionen (z.B. in dem Kinderlied „If you' re happy ${ }^{\text {"1 }}$ ) so faszinierend ist.

Hier fungiert die Musik nach Frohne-Hagemann und Pleß-Adamczyk (2005) als "Zeugin und emotionale Resonanzgeberin“, in welcher sie eine Haltefunktion hat, darüber hinaus jedoch auch die emotionale Resonanz des Individuums und dessen kulturellen Hintergrund widerspiegelt.

\subsection{Mentale Repräsentationen /Intermediärobjekte}

Als Übergangsraum beschreibt Winnicott (1979) einen „dritten Bereich [...], der weder im einzelnen noch in der äußeren Welt der erlebbaren Realität liegt. Dieser dritte Lebensbereich ist [...] durch ein schöpferisches Spannungsfeld gegeben“ (S. 127). An anderer Stelle heißt es: „Der intermediäre Bereich, von dem ich hier spreche, ist jener Bereich, der dem Kind zwischen primärer Kreativität und auf Realitätsprüfung beruhender, objektiver Wahrnehmung zugestanden wird. [...] Diese (Übergangsphänomene; T.S.) geben jedem Menschen, was stets für ihn Bedeutung behalten wird: einen neutralen Erfahrungsbereich, der nicht in Frage gestellt wird“ (Winnicott 1979, S. 21ff). Musik im Allgemeinen und Kinderlieder im Speziellen können in besonderer Weise diese Funktion vermitteln. So ist beispielsweise das Gute-Nacht-Lied ein verbindendes Drittes und vermittelt „Gemeinsamkeit im Sinne eines geteilten Interesses, worüber Intersubjektivität im Sinne Sterns erfahren wird und schließlich auch direkte zwischenmenschliche Begegnungen möglich werden" (Frohne-Hagemann u. Pleß-Adamczyk 2005; S. 101). Lieder können auch als Übergangsobjekte dienen, indem sie den Übergang zwischen Selbst und Nicht-Selbst herstellen (z.B. das „Pfeifen im Walde“). Diese Übergangsräume im Sinne Winnicotts und die damit verbundenen Erfahrungen der frühen Kindheit sind für den Beginn einer Beziehung zwischen Kind und Welt notwendig. Lempp (2003) spricht von einer „Art kopernikanischen Wende in der psychische Entwicklung“, wenn das Kind schließlich den Egozentrismus überwinden lernt und die Fähigkeit entwickelt, Gedanken und Gefühle anderer zu erkennen und von den eigenen zu unterscheiden. Diese auch als „cognitive perspective taking“ oder "theory of mind“ bezeichnete Fähigkeit wird im Spiel und durch das Spielen erworben, wobei z.B. Spiel- und Märchenlieder ein hilfreiches mentales Gerüst bieten können.

\footnotetext{
${ }^{1}$ Deutsche Version von G. Schöne: „Wenn du glücklich bist, dann klatsche in die Hand (klatschen). Zeig mir, wenn du bei mir bist, wie dir so zumute ist und wenn du glücklich bist, dann klatsche in die Hand. / Wenn du wütend bist, dann stampfe mit dem Fuß.../ Wenn du traurig bist, dann seufze doch einmal (aach)..." etc.
}

(C) Vandenhoeck \& Ruprecht GmbH \& Co. KG, Göttingen 


\subsection{Motivation und Intention}

Nach Holodynski und Oerter (2002) geht es bei dem Begriff Motivation um eine Beschreibung und Erklärung dafür, warum wir uns bewegen und bewegen lassen. Zu den positiven Motivationen zählen Exploration (Neugier), Erfolgsorientierung (Streben) und Sehnsucht nach Nähe (Bindungsbedürfnis), während negative Motivationen der Reduktion bzw. Vermeidung von Schmerz oder irritierender Anspannung dienen (Bucci 1997; zitiert nach Resch et al. 1999). Daraus ergibt sich, dass Motivation eng mit dem Belohnungssystem und dem Neurotransmitter Dopamin verknüpft ist. Außer durch z.B. Kokain, Schokolade und Blickkontakt (Spitzer 2002a) wird das Belohnungssystem auch durch positiv konnotierte Musik aktiviert, wie Blood und Zatorre (2001) in einer Bildgebungsstudie nachweisen konnten. Musik wirkt dabei in doppelter Weise: Sie stimuliert das körpereigene Belohnungssystem, wodurch die Ausschüttung von Dopamin (aus Neuronen der Area A 10 in den Nucleus accumbens) und von endogenen Opioiden (aus Neuronen des Nucleus accumbens in weite Teile des Frontalhirns) ausgelöst wird, und sie bewirkt eine Reduktion der Aktivität in Hirnstrukturen, die bei Angst (Amygdala) oder unangenehmen Erfahrungen (ventromedialer präfrontaler Kortex) aktiviert werden. Dies mag veranschaulichen, dass Musik sowohl als Trostlied („Heile, heile Segen“) als auch als Schlachtlied oder in weniger martialischer Form als Hymne vor einem Fußballländerspiel eine anxiolytische und motivierende Wirkung hervorruft.

Zusammenfassend lässt sich die entwicklungspsychologische Bedeutung von Musik und (Kinder-)Liedern sowohl von analytischen als auch neurobiologischen Theorien ableiten und erklären. Kritisch muss angemerkt werden, dass die Bezüge zu den verschiedenen Domänen der basalen psychischen Funktionen unterschiedlich intensiv untersucht sind und es daher weiterer Forschungsbemühungen in diesen Bereichen bedarf, um diese Zusammenhänge besser zu verstehen. Solche Anstrengungen könnten sich sowohl für die Musik- als auch für die Entwicklungspsychologie als fruchtbar erweisen.

\section{Lieder in der Kindermusiktherapie}

Art und Ausmaß des Einsatzes von Liedern in der musiktherapeutischen Arbeit sind vielfältig und hängen in erster Linie von der Persönlichkeit des Musiktherapeuten, seiner Ausbildung und Ausrichtung (z.B. anthroposophisch vs. analytisch orientiert), vom Setting (z.B. Einzel- oder Gruppentherapie) sowie vom Alter und Störungsspektrum der Klientel ab. Unabhängig davon formulieren Plahl und KochTemming (2005) eine Reihe von Indikationen und Zielsetzungen für den Einsatz von Liedern (S. 181):

(C) Vandenhoeck \& Ruprecht GmbH \& Co. KG, Göttingen 
- zur Strukturgebung,

- zur Kontaktaufnahme und zur Beziehungsregulation,

- zur Aufmerksamkeitsfokussierung und Aktivierung,

- zur Entspannung, Lockerung und Regression,

- zur Förderung einzelner Entwicklungsbereiche (insbesondere Motorik, Wahrnehmung, Sprache und Sozialverhalten),

- zum Ausdruck von Gefühlen und Bedürfnissen und

- zur Verarbeitung emotional belastender Erlebnisse und Zustände.

\subsection{Klassische/aktuelle Kinderlieder}

Bei Decker-Voigt (1999) heißt es: „Die ständige Wiederholungsfreude des Kindes (übrigens auch des Kindes in uns Erwachsenen) an Musik beruht darauf, dass es sich bei jeder Wiederholung mit dem einfachen Kinderlied zu identifizieren vermag: Das Lied wird zur Strukturierungshilfe in dieser ständig neuen Welt, denn in der Begeisterung der Wiedererkennung seiner Musik trennt das Kind nicht mehr zwischen sich und dem Gehörten, sondern ist Teil davon" (S. 203f.). So sind klassische und aktuelle Kinderlieder nicht nur Teil des kulturellen Erbes, sondern gehören zum Repertoire eines jeden $\mathrm{Mu}$ siktherapeuten. „Schon kleinere Kinder kennen mitunter altertümliche Kinderlieder, die kaum in breiterem rezenten Gebrauch stehen, dafür aber dem individuellen Liedbesitz von Eltern, Großeltern oder Verwandten entspringen und so weitergetragen werden" schreibt Freitag (2001, S. 36), wenngleich konstatiert werden muss, dass diese Form der Überlieferung eher rückläufig scheint. Dabei ist gerade das von der Mutter gesungene (Kinder-)Lied im besonderen Maße von einer individuellen Qualität, welches wie eine vokale Signatur, wie ein akustisches Erkennungszeichen fungiert (Bergeson u. Trehub 2002), was wiederum zurückverweist auf die emotionale Bedeutung (s.o.). Neben Kindergarten, Schule und Freizeitgruppen sind es aber heutzutage vor allem Bild- und Tonmedien, über die Liedgut und Musik an Kinder vermittelt wird.

Eine kleine Szene aus einer Musiktherapie mit einer Kindergruppe in der Kinderund Jugendpsychiatrie mag veranschaulichen, in welcher Weise sich durch ein allen bekanntes Kinderlied eine Beziehung und Interaktion herstellen lässt: Während die Co-Therapeutin der 8-jährigen Lisa auf deren Bitten hin „Bruder Jakob“ in verschiedenen Sprachen vorsingt, greift der 11-jährige Bastian, der vorher versucht hatte, auf der Gitarre „Lady in Black“"zu spielen, dieses Lied auf. Mit Hilfe des Musiktherapeuten gelingt ihm eine Gitarrenbegleitung zu dem Kanon, der quer durch den Raum einen ersten Moment von Gemeinsamkeit und Aufeinander-Bezogenheit entstehen lässt.

\subsection{Kontrafaktur}

Unter Kontrafaktur wird im Allgemeinen die geistliche Nachdichtung eines weltlichen Liedes - und vice versa - verstanden, wobei die Melodie zwar dem neuen Text angepasst wird, im Wesentlichen jedoch erhalten bleibt. Auf diese Weise las-

(C) Vandenhoeck \& Ruprecht GmbH \& Co. KG, Göttingen 
sen sich verschiedenste bekannte Lieder - sei es aus dem Volks- und Kinderliedbereich oder aus der Popularmusik - umtexten und somit der jeweiligen therapeutischen Situation entsprechend gestalten. Dies kann rein situativ und improvisierend geschehen oder als ausgearbeitete Umdichtung durch den Therapeuten und/oder den Patienten. Letztere Form soll anhand eines Beispiels aus der Musiktherapie mit krebskranken Kindern dargestellt werden. Musiktherapie in der Kinderonkologie nimmt in vielfacher Hinsicht eine Sonderstellung ein (Klientel, Setting, Therapieziele etc.), auf die in diesem Rahmen nicht näher eingegangen werden kann. Musikpsychotherapie wird dabei nicht als „alternative“ oder „sanfte“ Behandlungsmethode verstanden, die beispielsweise eine Chemotherapie ersetzen könnte. Wie Grießmeier und Bossinger (1994) betonen, kann Musiktherapie zwar den Krebs nicht heilen, aber dabei helfen, mit dem Krebs zu leben, was zu einer Verbesserung der Lebensqualität führt.

In diesem Zusammenhang geht es aus therapeutischer Sicht auch um die Wahrnehmung, Bearbeitung und Integration aggressiver Impulse, wie sie bei den Patienten in Reaktion auf die Erkrankung und die damit verbundenen invasiven medizinischen Maßnahmen entstehen. „Musiktherapie hilft den Kindern, besser mit den seelischen Folgen der Krankheit zu leben, indem sie die Kinder ermutigt, auch negative Gefühle und Empfindungen, die die Krankheit bei ihnen auslöst, zuzulassen; (...) indem sie hilft, unangenehme Behandlungsschritte ,in Szene zu setzen', darüber zu reden und so die Bedrohlichkeit zu vermindern" (Grießmeier u. Bossinger 1994; S. 170). Die folgende Umdichtung (mündliche Überlieferung; persönliche Mitteilung von G. Kappelhoff) von „Die Affen rasen durch den Wald“ zeigt in humorvoller Weise (im Übrigen auch ein wichtiger salutogenetischer Faktor; s. Freud 1905, S. 260ff.), wie die Wendung ins Aktive durch Musik gelingen kann:

Die Ärzte rasen durch den Flur, sie fragen sich, wer war das nur? Die ganze Klinikbande brüllt: Wo ist das Röntgenbild, wo ist das Röntgenbild, wer hat das Röntgenbild geklaut?

Der Doktor Müller, welch ein Graus, reißt sich die Haare einzeln aus. Die ganze Klinikbande brüllt: Wo ist das Röntgenbild...

Professor Meyer steht am Schrank, sie ist vor Ärger schon ganz krank. Die ganze Klinikbande brüllt: Wo ist das Röntgenbild... 
Die Visite steht verzweifelt da wie schön das Röntgenbild doch war...

Die ganze Klinikbande brüllt:

Wo ist das Röntgenbild...

(usw.)

Am Ende des Liedes ist es natürlich die Patientin selbst, die das Röntgenbild in Besitz genommen hat:

Die Laura an zu lachen fängt, hat's Bild im Zimmer aufgehängt.

Die ganze Klinikbande brüllt:

Da ist das Röntgenbild, da ist das Röntgenbild, sie hat das Röntgenbild geklaut!

\subsection{Spiellieder}

„Psychotherapie geschieht dort, wo zwei Bereiche des Spielens sich überschneiden: der des Patienten und der des Therapeuten. Psychotherapie hat mit zwei Menschen zu tun, die miteinander spielen. Hieraus folgt, dass die Arbeit des Therapeuten dort, wo Spiel nicht möglich ist, darauf ausgerichtet ist, den Patienten aus einem Zustand, in dem er nicht spielen kann, in einen Zustand zu bringen, in dem er zu spielen imstande ist." (Winnicott 1979; S. 90)

Musik ist in allererster Linie Spiel - nicht umsonst trägt eine an Einfallsreichtum und Kreativität kaum zu überbietende Einspielung der bekannten Jazz-Musiker Bobby McFerrin und Chick Corea den schlichten Titel „Play“. Laut Oerter (1997) genügt Musizieren den typischen Merkmalen des Spiels und kann im Wesentlichen mit drei Spielformen in Beziehung gebracht werden: dem sensomotorischen Spiel, dem Konstruktionsspiel und dem Regelspiel. Anschließend an das obige Zitat von Winnicott mag es kaum verwundern, dass auch und gerade in der Musiktherapie dem Spiel des Kindes eine große Bedeutung zukommt. Die Übergänge sind dabei fließend, sodass „Zwischen Spielen und Musikmachen keine klare Trennlinie gezogen werden kann“ (Petersen u. Thiel 2001). Insbesondere Lieder laden dazu ein, Text und Musik mit Bewegungen, Tanz oder szenischem Spiel zu verbinden. Mahns (1997) definiert Spiellieder als eine Liedgattung, deren Hauptakzent auf dem Wort „Spiel“ liegt und deren Inhalt auf der Phantasieebene angesiedelt ist: „Im Umgang mit Spielliedern und Musik kann sich das Spielverhalten in entsprechend angstfreier Atmosphäre zu regressivem Verhalten verändern. Die Kinder können ihren Bedürfnissen nachgehen, träumen, regredieren, sich regenerieren und neue Handlungsmöglichkeiten erproben. Die einzelnen Kinder werden an der Gestaltung des Liedes beteiligt und können es auf ihre Weise verändern und inszenieren, sei es durch Bewegung, $\mathrm{Mu}$ sikinstrumente oder durch neue Text- und Strophenideen“ (S. 90).

(c) Vandenhoeck \& Ruprecht GmbH \& Co. KG, Göttingen 
Das folgende Spiellied „Schiffbruch“ stammt aus einer Gruppen-Musiktherapie, in der die Kinder in der vorherigen Stunde von sich aus begonnen hatten, sich Höhlen zu bauen und sich darin zu verstecken. Die hier dargestellte Komposition sollte daran anknüpfend das Thema des „sicheren Ortes“ (Reddemann 2004) aufnehmen und den Patienten einen spielerischen Umgang erlauben. Das Lied bildete dabei einen musikalischen Rahmen für ein Fangen-Spiel, das an den so genannten StoppTanz angelehnt war. D.h. die Kinder bewegten sich frei im Raum, solange der Therapeut das Lied sang und spielte. Unterbrach dieser sein Spiel, mussten die Kinder zu ihrer Ausgangsbasis zurückkehren, um nicht von Piraten (Co-Therapeutin und Betreuerin) gefangen zu werden. Wer doch gefangen wurde, konnte sich durch das Erraten des Klanges eines Musikinstruments wieder befreien.

Schiffbruch - doch Land in Sicht!

Zur Ostereierinsel, so weit ist das nicht.

Doch um dahin zu geraten -

Vorsicht vor Piraten -

Müsst ihr drei Mal richtig raten...

\subsection{Situationslieder}

Situationslieder beziehen sich, wie Plahl und Koch-Temming (2005) ausführen, „direkt auf das aktuelle therapeutische Geschehen und werden von der Therapeutin spontan für das Kind, gemeinsam mit dem Kind oder vom Kind selbst in der Situation erfunden“ (S. 180). Je nach Therapieausrichtung und Störungsbild stellen von therapeutischer Seite eingebrachte Situationslieder einen wesentlichen Bestandteil der Beziehungs- und Kommunikationsgestaltung dar: „Durch den auf die Situation bezogenen Text kann die Musiktherapeutin das Verhalten des Kindes spielerisch aufgreifen und dadurch eine Stimmungsänderung bewirken“" (Plahl u. Koch-Temming 2005; S. 180). Aber auch „Lieder ohne Worte“ - vom Therapeuten gesummt oder gesungen - können in besonderen Phasen oder Situationen während der Behandlung indiziert sein. Häufig werden Situationslieder instrumental begleitet, wozu sich Harmonieinstrumente wie Klavier, Gitarre oder Akkordeon besonders eignen. Auch Orff- und Rhythmusinstrumente können zur Begleitung eingesetzt werden, erlauben unter Umständen auch leichter eine Einbeziehung oder ein „Einfädeln“ des Kindes. Schumacher und Calvet-Kruppa (2005) beschreiben den Einsatz von Situationsliedern im Sinne einer Krisenintervention bei affektiven Ausnahmezuständen: „Die musikalische Improvisation (meine Stimme, unser instrumentales Spiel sowie auch ein die Situation beschreibender Text eines improvisierten Liedes) wird dynamisch und rhythmisch genau auf die affektiven Äußerungen des Kindes abgestimmt und formal gestaltet" (Schumacher u. Calvet-Kruppa 2005, S. 290). Die Autorinnen weisen abschließend darauf hin, dass Situationslieder mit beschreibendem Text nur sinnvoll seien, „wenn der Affekt sei-

(C) Vandenhoeck \& Ruprecht GmbH \& Co. KG, Göttingen 
nen Höhepunkt noch nicht erreicht oder schon überschritten hat" (Schumacher u. Calvet-Kruppa 2005, S. 290).

In ihrer entwicklungspsychologisch fundierten Musiktherapie (Schumacher u. Calvet-Kruppa 2005) stellt die Arbeit mit Kindern mit einer Störung aus dem autistischen Spektrum einen langjährigen Schwerpunkt dar. Die besondere Herausforderung im Kontakt mit Patienten mit dieser Wahrnehmungsverarbeitungsstörung liegt im Ausbalancieren von Nähe und Distanz: „Musiktherapie muss daher zunächst eine möglicherweise gestörte Wahrnehmung berücksichtigen. Methodisch muss sie ganz vom ,So-Zustand' des Kindes, das heißt von den Reizen, die das Kind sich selbst verschafft, ausgehen“ (Schumacher u. Calvet-Kruppa 2005; S. 287). Den Einsatz von Situationsliedern beschreibt Schumacher (1994) in „Max - eine Fallgeschichte“: „Ich besinge, was er tut, nehme den sichtbaren Rhythmus seiner Bewegungen auf, entferne mich aber immer weiter, indem ich frei improvisiere und damit einen Klangraum schaffe, der nicht direkt mit ihm und seinem Tun zusammenhängt" (Schumacher 1994, S. 37). Diese Art der Interaktion erlaubt bereits in der zweiten Stunde eine „erste Spielform“, die von Schumacher (1994) folgendermaßen protokolliert wird:

„Max setzt sich auf die große Doppelfelltrommel und ich spiele einen ratternden Zugrhythmus auf dem eisernen Rand der Trommel in Kombination mit dem Fell, auf dem er sitzt, wobei er die dabei entstehende Vibration zu spüren bekommt. Ich singe: ,Der Max, der Max fährt mit dem Zug, die Karin, die Karin fährt auch mit dem Zug. Es geht dahin durch Wald und Feld, es geht dahin, dahin, dahin.... Maxens Mimik zeigt, dass er zuhört und aufnimmt. Er steht genau an Ende des Liedes auf und kehrt zur Trieselbewegung ${ }^{2}$ zurück, die ich als Zwischenspiel in das Zuglied einbaue" (Schumacher 1994, S. 38).

Eine andere Form des Situationsliedes sind Lieder, die spontan vom Kind selbst in die Therapie eingebracht werden. Freitag (2001) schreibt: „Das Spontansingen kleiner Kinder lässt Rückschlüsse zu auf die Lebendigkeit und Unbefangenheit familiären Musikmachens“ (S. 36). Um wie viel mehr gilt diese Aussage für das sich Widerspiegeln psychisch belastender Lebenssituationen in Text und Musik im therapeutischen Setting. In einer Phase einer längeren ambulanten Therapie mit einem 7-jährigen Mädchen (Leni), das sich wegen einer emotionalen Störung in Behandlung befand, hatte sich ein Ritual herausgebildet:

Leni bat immer am Ende der Stunde darum, ein bestimmtes Lied („Wenn der Sommer kommt"von Rolf Zuckowski) vorgespielt zu bekommen, das ich textlich je nach Stundenverlauf oder anliegenden Ereignissen adaptierte. In einer Stunde überraschte mich Leni damit, dass sie selbst ein Lied „mitgebracht“ habe, welches sie mir vorsingen wolle:

\footnotetext{
${ }^{2}$ Trieseln $=$ etwas durch die Finger rieseln lassen (Schumacher 1994).

(c) Vandenhoeck \& Ruprecht GmbH \& Co. KG, Göttingen
} 
Wir fahren mit der Kutsch, ja, ja.

Wo soll'n wir nur hinfahr'n?

$\mathrm{Zu}$ der lieben Großmama,

da wollen wir nun hin mal fahr'n.

$\mathrm{Oh}$, oh, oh - uns're Kohle ist nun leer,

müssen wir wohl neue hol'n.

Ich geh' schon, du bleibst nun hier.

Miau, miau, miau! Habt ihr mich vergessen?

Ich muss doch auch noch mit! Wau, wau, wau!

Habt ihr mich auch vergessen? Nein, nein, nein!

Steig schnell ein und dann geht's weiter...

Musikalisch, aber auch inhaltlich, knüpft dieses Ex-Tempore-Lied an den Song „Old McDonald had a farm“ an, das wir zu Beginn jener Therapiestunde gemeinsam gesungen hatten. Im Text klingen als Themen sowohl Parentifizierung („Ich geh' schon, du bleibst nun hier") als auch starke Verlassensängste an („Habt ihr mich vergessen? Ich muss doch auch noch mit!“), wie sie für ein Kind von zwei suchtkranken Eltern (der Vater war an Komplikationen wenige Jahre zuvor verstorben) nur allzu gut nachvollziehbar sind. Auf weitere Deutungsebenen (aktueller Bezug, intrapsychisches Erleben und Triebprozesse sowie die therapeutische Beziehung) kann an dieser Stelle nicht eingegangen werden. Das Beispiel soll lediglich belegen, wie reichhaltig das Material sein kann, das durch Situationslieder vom Kind selbst in die Therapie eingebracht wird.

\subsection{Begrüßungs- und Abschiedslieder (Gruppentherapie)}

Laut Rösing und Barber-Kersovan (1997) werden mit dem vieldeutigen Begriff „Ritual“ Handlungsabläufe bezeichnet, die sich nach einem „vorgegebenen Muster“ wiederholen. Ursprünglich aus dem religiösen Kontext stammend, können unter diesem Begriff „Handlungen mit einem Symbolwert“ verstanden werden, die von einer Gruppe als wesentlich angesehen werden: „Dem Ritual kommt die Funktion einer nonverbalen Gruppenkommunikation zu“ (S. 136).

Somit eignet sich Musik zum einen als Träger ritualisierter Handlungen, zum anderen erklärt sich aus dieser phylogenetisch originären Einsatzform der Musik auch deren ontogenetische Bedeutung, welche bereits intrauterin beginnt. Hierauf weist Decker-Voigt (1999) hin, wenn er im Zusammenhang mit pränataler Einführung von musikalischen Strukturierungshilfen schreibt: „Ein Ritual hat etwas mit Wiederkehr zu tun, was wiederum Sicherheit vermittelt und Struktur“ (S. 52). Daher mag es kaum verwundern, dass in den meisten Kindermusiktherapien Begrüßungsund Abschiedslieder zur Umrahmung der Therapiestunde eingesetzt werden. Solche Rituale geben dem Kind Sicherheit und Orientierung und fördern Gemeinsamkeit (Plahl u. Koch-Temming 2005). Sie dienen sowohl dem Containing als auch der

(C) Vandenhoeck \& Ruprecht GmbH \& Co. KG, Göttingen 
Förderung eines Selbstwirksamkeitserlebens, in dem Kinder schon nach kurzer Zeit ein positives Selbstwertgefühl erfahren, etwas sicher zu können bzw. zu wissen (Klöppel u. Vliex 2004).

Der Veranschaulichung sollen ein Begrüßungs- und ein Abschiedslied aus der eigenen Arbeit dienen. Im ersten Beispiel wurden die Namen der Patienten geändert.

Hey Lisa, Tim und Bastian und Adrian, ihr seid hier - (wir sind hier) alle vier - (alle vier) und wir machen jetzt Musik (Klatschen) und wir hör'n einander zu (,pschschscht“) und wir machen jetzt Musik (Klatschen) weil wir heut zusammen sind.

Das Begrüßungslied erhält in dreifacher Hinsicht eine besondere Valenz: erstens durch die exponierte Stellung zu Beginn der jeweiligen Stunde, zweitens durch die Personalisierung und die direkte namentliche Ansprache der Kinder, die drittens eine positive Wertschätzung darüber erleben, dass jemand speziell für sie ein Lied gefertigt hat. Des Weiteren beinhaltet das Lied einen Vorgriff auf wesentliche Elemente und Anforderungen der Therapiesitzung, nämlich den Wechsel zwischen gemeinsamem Musizieren auf der einen und dem Zuhören- und Abwarten-Können auf der anderen Seite.

Beschlossen wurde die Musiktherapiestunde immer mit dem folgenden Abschiedslied (Text: V. Stein; Musik: Stein/Stegemann):

Ciao, ciao und Good bye,

die Musik ist gleich vorbei.

Ciao, ciao und Good bye,

es ist jetzt gleich halb zwei.

Ein ander' Mal am selben Ort

setzen wir das ganze fort.

Heute ham wir Krach gemacht, war'n leise auch wie in der Nacht

In der Musik finden sich sowohl das Betrübt-Sein darüber, dass etwas Schönes zu Ende geht, als auch die Vorfreude auf ein nächstes Mal, auf die Fortsetzung, wieder. Auch die Pole zwischen Musikmachen und Stille aus dem Begrüßungslied werden hier wieder aufgegriffen. Die Bedeutung ritualisierter Elemente wird u.a. dadurch dokumentiert, dass Kinder besonderen Wert auf diese Rituale legen und sie deren Einhaltung regelrecht einfordern.

(c) Vandenhoeck \& Ruprecht GmbH \& Co. KG, Göttingen 


\subsection{Semistrukturierte Lieder - Das 3-Dinge-Lied}

Angelehnt an die klassische Interviewfrage „Welche drei Dinge würden Sie mit auf eine einsame Insel nehmen?" sowie an bekannte projektive Testverfahren wie das „10-Wünsche-Phantasiespiel“ (Klosinski 1988) stellt das folgende Beispiel eine Liedform dar, die ich als „semistrukturiert“ bezeichnen möchte. Sie ist darauf angelegt, dem Kind die Möglichkeit zu geben, sich niedrigschwellig an der Gestaltung eines Liedes zu beteiligen. Gleichzeitig kann diese Liedform als projektives diagnostisches Verfahren angewendet werden. Im Rahmen der Gruppe bietet dies die Besonderheit, dass die Wünsche geteilt werden und von den Mitpatienten und Therapeuten durch die entsprechenden Lautäußerungen (mmmhh, ooohhh und aaahhh) positiv konnotiert werden. Dies fördert den Gruppenzusammenhalt sowie die Achtung vor Bedürfnissen und Anliegen der anderen.

Eins - zwei - drei - was hast du dabei?

One - two - three - das errätst du nie!

Bir - iki - üç - sei doch nicht so müksch!

Acht - neun - zehn - na gut, mal seh'n:

(gesprochen) Also, ich nehme mit:

1............... (Alle: $\mathrm{mmmhhh!)}$

2. .............. (Alle: ooohhh!)

3. ............. (Alle: aaahhh!)

Das ist echt der Hit! Das nimm mal mit!

Der 10-jährige Bastian aus der bereits oben erwähnten Kindermusiktherapiegruppe war mit einer Störung des Sozialverhaltens vor dem Hintergrund eines massiven „Rosenkrieges" der bereits geschiedenen Eltern in stationärer Behandlung. Er wünschte sich in der letzten Musiktherapiestunde „ein Zelt, einen Elefanten und eine MP“. Das Zelt dürfte Ausdruck für den Wunsch nach einem „eigenen“ Zuhause und einem sicheren Ort sein. Auf der anderen Seite drücken sich in der Maschinenpistole („MP“) die aggressive Komponente aus, aber auch das Bedürfnis, sich schützen zu können. Darüber, wofür der „Elefant“ steht, lässt sich zunächst nur spekulieren (z.B. lautet ein afrikanisches Sprichwort: „Wo sich die Elefanten streiten, gehen die Blumen kaputt!“).

\section{7 "Die alte Moorhexe"}

„Eine Hexe, die aus den Angstphantasien eines Kindes geboren ist, wird es verfolgen; aber eine Hexe, die man in ihren eigenen Ofen stoßen und verbrennen kann, ist eine Hexe, von der das Kind sich befreit glauben kann."

(Bruno Bettelheim, Kinder brauchen Märchen, 1977; S. 157)

(C) Vandenhoeck \& Ruprecht GmbH \& Co. KG, Göttingen 
Zum Abschluss soll an dem Lied „Die alte Moorhexe“ von Wolfgang Jehn gezeigt werden, wie unterschiedlich ein Musikstück im musiktherapeutischen Kontext eingesetzt werden kann. Im ersten Fall kommt das Lied als Kontrafaktur in der Kinderonkologie zum Einsatz. Die Funktion entspricht dabei der bereits unter 2.2. beschriebenen.

Aus der CD „When sunshine gets cold“, Wolfgang Köster, Münster, 1998:

Die dicke Moorhexe hext, hext im Krankenhaus herum, dreht sich wild im Tanz herum, lacht sich schief und lacht sich krumm, wenn die Kinder ängstlich zittern und die Mütter noch mehr bibbern, kommt sie schnell hereingebraust - um die Ecke gesaust.

Uahuahuahuu...

Die Hexe springt und sie faucht um die Mutter wild herum, und die Mutter rollt vor Schreck mit dem Krankenhausbett weg, und das Kind guckt aus dem Fenster, lacht so laut, dass die Gespenster alle einen Schrecken kriegen und schnell in die Lüfte fliegen.

Und dann fliegt die Hexe schnell ins Schwesternzimmer mit Gebrüll, so dass die Schwestern voller Schreck ihre Spritzen werfen weg, und die Sonja sowieso kriegt die Spritze in den Po, Dr. H. lacht sich krumm, ist er schlau oder dumm?

Und es dauert nicht sehr lang, sieht wie ne Spritze fliegen kann Dr. H. ins Gesicht, dass er sich ganz schnell erbricht. Und dann hat er nichts zu lachen, kann auch keine Späße machen, seine Hose ist ganz voll, und das findet er nicht toll.

Professor J. dieser Knilch trinkt am Tag zehn Liter Milch. Und er sagt, dass keine Frage, Carolin bleibt noch zehn Tage Muss ich mir das gefallen lassen, der hat wohl nicht mehr alle Tassen in dem großen Küchenschrank, ist er gesund oder krank?

Oh Gott, oh Gott, ihr armen Ärzte, das sind doch nur alles Scherze, nehmt mir das bloß ja nicht übel, sonst versteck ich mich im Kübel und dann schäm ich mich ganz doll, doch ich weiß nicht, was das soll, lasst uns alle Frieden schließen, sonst werden meine Tränen fließen.

In noch drastischerer Weise als im Lied vom „Röntgenbild“ werden hier die Alltagsthemen einer Kinderkrebsstation aufgegriffen. Schmerzen und Todesangst treten allegorisch in Gestalt der Hexe und der Gespenster auf. Auch hier finden wir wieder die Wendung ins Aktive sowie den (schwarzen) Humor als Schlüssel für ein erfolgreiches Coping. 
Das zweite Beispiel stammt aus einer Gruppenmusiktherapie in einer Sprachheilschule (Mahns 1997). In diesem Kontext einer musiktherapeutischen Arbeit mit vier verhaltensauffälligen Jungen hatte die Musiktherapeutin das Lied der „alten Moorhexe“ eingeführt als Identifikationsangebot in Richtung auf das „böse Weibliche“ und das „gute Männliche“. Dazu schreibt die Autorin weiter: „Die Geschichte von der alten Moorhexe würde den unterschiedlichen Persönlichkeiten dieser Gruppe genügend Phantasieanregung, Spielraum und Identifikationsmöglichkeiten zu bieten haben" (dies., S. 55).

Während es im ersten Beispiel in erster Linie um das Lied, dessen Umtextung, Gestaltung und schließlich auch um die Tonaufnahme ging, bildet das Lied in seiner Ursprungsform im zweiten Fall lediglich das Ausgangsmaterial: „Die von mir angebotene Geschichte über die Moorhexe wird mit Interesse und Spannung verfolgt. Wir spielen gruselige Musik und versuchen, die Stimmung des Teufelsmoores einzufangen. Das Lied hat dabei nur nebensächlichen Charakter, und ich singe es im Wesentlichen als Strukturierungshilfe für die weiteren Phantasien“ (dies., S. 59). So entwickelt sich aus diesem Lied und der Geschichte ein Themenrahmen für die nächsten Stunden, in dem Triebkräfte durch Gruseln, Spuk und Zaubermacht ihren Platz finden. Die Jungen, im Alter zwischen acht und zehn Jahren, gehen der „alten Moorhexe“ zunächst als Zaubergehilfen zur Hand, verbünden sich aber schließlich gegen ihre Meisterin und hecken allerlei gegen diese aus, was zu guter Letzt in einer „Hexenverbrennung“ endet. Mahns resümiert: „Das von mir kommende Angebot der , alten Moorhexe’ bietet die Möglichkeit, die Aggressionen auf die Therapeutin zu richten. Darüber hinaus sind sexuelle Wünsche und Phantasien durch die symbolische Darstellung der Geschichte im Text selbst legitimiert. Das Angebot ermöglicht den Kindern, sich als Gruppe zu solidarisieren und ihre negativen Übertragungsgefühle im Spiel auf mich zu richten. Die schrittweise Entmachtung der Hexe durch die Hexengehilfen deutet darauf hin, wie sehr die Kinder unter ihrer eigenen oft machtlosen Position zu leiden haben. Ich meine auch den Wunsch zu spüren, man solle ihre angehende Männlichkeit wahr- und ernstnehmen. Gleichzeitig zeigten sie hier auch die enormen Bedürfnisse nach Autonomie und Freiheit" (dies., S. 64).

Diese beiden Fallvignetten sollen deutlich machen, wie viel Identifikations- und Interpretationsspielraum Kinderlieder bieten und auf welch unterschiedliche Weise sie sich dem Setting und der Zielsetzung entsprechend psychotherapeutisch einsetzen lassen.

\section{Coda}

Das Spektrum der Einsatzmöglichkeiten von Liedern in der musiktherapeutischen Arbeit mit Kindern spiegelt den Reichtum des kreativen Potentials von Musik und Poesie im Allgemeinen wider. Bedeutsam für die „richtige Anwendung“ ist zum

(C) Vandenhoeck \& Ruprecht GmbH \& Co. KG, Göttingen 
einen - wie in jeder anderen Psychotherapie auch - das Gespür für den passenden Moment, das genaue Timing sowie die entsprechende Dosierung. Voraussetzung dafür ist einerseits ein umfangreiches und stilübergreifendes Repertoire an Liedern, verbunden mit der Fähigkeit - sowohl musikalisch als auch textlich - flexibel und improvisatorisch geschult auf die jeweiligen situativen und individuellen Bedürfnisse eingehen zu können. Somit lassen sich in der Musik der Wiedererkennungseffekt mit den damit einhergehenden Assoziationen sowie das aus der Situation und der Beziehung entstehende Einzigartige und Nicht-Wiederholbare des Augenblicks vereinen. Darin liegt die besondere Kraft und Chance im therapeutischen Einsatz von Liedern. Auf diese Weise können Lieder in einer ganzen Reihe von therapeutisch relevanten Funktionen Eingang in die musiktherapeutische Behandlung finden, wie es von Frohne-Hagemann und Pleß-Adamczyk (2005) beschrieben wird: „Musik kann in der Therapie mehrere therapeutische Funktionen gleichzeitig und dabei jeweils verschiedene Facetten haben“ (S. 95). Diese von den Autoren definierten Funktionen reichen von Musik als basalem Sinnesstimulus über die Containerfunktion hin bis zur Musik als „Ressource, Schutzfaktor und Nährsubstanz" (Frohne-Hagemann u. Pleß-Adamczyk 2005, S.102). Das Lied nimmt insofern eine Sonderstellung ein, als dass die Musik in diesem Kontext nicht nur „Repräsentation und Symbol“ (Frohne-Hagemann u. Pleß-Adamczyk 2005, S. 95) ist, sondern gleichsam eine Trägersubstanz für das Wort und seine potentiell heilende Wirkung.

\section{Literatur}

Bastian, H. G. (2002): Musik(erziehung) und ihre Wirkung. Eine Langzeitstudie an Berliner Grundschulen. (2. Ausg.). Mainz: Schott.

Bergeson, T. R.; Trehub, S. E. (2002): Absolute pitch and tempo in mother's songs to infants. Psychol. Sci. 13: 72-75.

Bettelheim, B. (1977): Kinder brauchen Märchen. Stuttgart: Deutsche Verlags-Anstalt.

Blood, A. J.; Zatorre, R. J. (2001): Intensely pleasurable responses to music correlate with activity in brain regions implicated in reward and emotion. PNAS 98: 11818-11823.

Bruhn, H.; Oerter, R. (1997): Entwicklung grundlegender musikalischer Fähigkeiten. Die ersten Lebensmonate. In H. Bruhn, R. Oerter; H. Rösing (Hrsg.), Musikpsychologie. Ein Handbuch. Hamburg: Rowohlt.

Bucci, W. (1997): Psychoanalysis and Cognitive Sciences. A Multiple Code Theory. New York: Guilford.

Decker-Voigt, H.-H. (1999): Mit Musik ins Leben. Kreuzlingen: Ariston.

Doherty, C. P.; Fitzsimons, A.; Asenbauer, B.; Staunton, H. (1999): Discrimination of prosody and music by normal children. European Journal of Neurology 6: 221-226.

Erk, S., Kiefer, M.; Grothe, J.; Wunderlich, A. P.; Spitzer, M.; Walter, H. (2003): Emotional context modulates subsequent memory effect. NeuroImage 18: 439-447.

Fox, D. B. (1990): An analysis of the pitch characteristics of infant vocalization. Psychomusicology. Special Issue: Music Child Development 9: 21-30.

(c) Vandenhoeck \& Ruprecht GmbH \& Co. KG, Göttingen 
Freitag, T. (2001): Kinderlied - Von der Vielfalt einer musikalischen Liedgattung. Frankfurt a.M.: Peter Lang.

Freud, S. (1905): Der Witz und seine Beziehung zum Unbewussten. Frankfurt: Fischer.

Frohne-Hagemann, I.; Pleß-Adamczyk, H. (2005): Indikation Musiktherapie bei psychischen Problemen im Kindes- und Jugendalter. Göttingen: Vandenhoeck \& Ruprecht.

Grießmeier, B.; Bossinger, W. (1994): Musiktherapie mit krebskranken Kindern (Vol. 13). Stuttgart: Fischer.

Holodynski, M.; Oerter, R. (2002): Motivation, Emotion und Handlungsregulation. In R. Oerter \& L. Montada (Hrsg.), Entwicklungspsychologie. Weinheim: Beltz.

Klöppel, R.; Vliex, S. (2004): Helfen durch Rhythmik. Kassel: Gustav Bosse.

Klosinski, G. (1988): Das „10-Wünsche-Phantasiespiel”. Gedanken und Erfahrungen zum „projektiven Fragen” am Beginn des therapeutischen Dialoges mit Kindern und Jugendlichen. Acta Paedopsychiat. 51: 164-171.

Lempp, R. (2003): Das Kind im Menschen. Stuttgart: Klett-Cotta.

Mahns, B. (1997): Musiktherapie bei verhaltensauffälligen Kindern. Stuttgart: Fischer.

Oerter, R. (1997): Musik und Individuum. Handlungstheoretische Fundierung. In H. Bruhn, R. Oerter; H. Rösing (Hrsg.), Musikpsychologie. Ein Handbuch. Hamburg: Rowohlt.

Papoušek, M. (2003): Vorwort. In M. Nöcker-Ribaupierre (Hrsg.), Hören - Brücke ins Leben (pp. 7-11). Göttingen: Vandenhoeck \& Ruprecht.

Petersen, D.; Thiel, E. (2001): Tonarten, Spielarten, Eigenarten. Kreative Elemente in der Musiktherapie mit Kindern und Jugendlichen. Göttingen: Vandenhoeck \& Ruprecht.

Plahl, C.; Koch-Temming, H. (2005): Musiktherapie mit Kindern. Bern: Huber.

Rauscher, F. H.; Shaw, G. L. (1998): Key components of the Mozart effect. Perceptual and Motor Skills 86: 835-841.

Reddemann, L. (2004): Psychodynamisch Imaginative Traumatherapie. Stuttgart: Pfeiffer bei Klett-Cotta.

Resch, F.; Parzer, P.; Brunner, R. M.; Haffner, J.; Koch, E.; Oelkers, R.; et al. (1999): Entwicklungspsychopathologie des Kindes- und Jugendalters. Weinheim: PVU.

Rösing, H.; Barber-Kersovan, A. (1997): Sozialpsychologische Aspekte. Konzertbezogene Verhaltensrituale. In H. Bruhn; R. Oerter; H. Rösing (Hrsg.), Musikpsychologie. Ein Handbuch. Hamburg: Rowohlt.

Schumacher, K. (1994): Musiktherapie mit autistischen Kindern. Stuttgart: Fischer.

Schumacher, K.; Calvet-Kruppa, C. (2005): „Untersteh dich!” - Musiktherapie bei Kindern mit autistischem Syndrom. In C. Plahl; H. Koch-Temming (Hrsg.), Musiktherapie mit Kindern. Bern: Huber.

Spitzer, M. (2002a): Lernen. Heidelberg: Spektrum.

Spitzer, M. (2002b): Musik im Kopf. Stuttgart: Schattauer.

Welch, G. F. (2005): Singing as communication. In D. Miell, R. MacDonald; D. J. Hargreaves (Eds.), Musical Communication (pp. 239-259). New York: Oxford University Press.

Winnicott, D. W. (1979): Vom Spiel zur Kreativität (2. Ed.). Stuttgart: Klett-Cotta.

Korrespondenzadresse: Dr. med. Thomas Stegemann, Klinik für Psychiatrie und Psychotherapie des Kindes- und Jugendalters des Universitätsklinikums Hamburg, Martinistr. 52, 20246 Hamburg;

E-Mail: t.stegemann@uke.uni-hamburg.de

(C) Vandenhoeck \& Ruprecht GmbH \& Co. KG, Göttingen 